EESTI NSV TEADUSTE AKADEEMIA TOIMETISED. 25. KOIDE FUUSIKA * MATEMAATIKA. 1976, NR. 4

ИЗВЕСТИЯ АКАДЕМИИ НАУК ЭСТОНСКОИ ССР. ТОМ 25 ФИЗИКА * МАТЕМАТИКА. 1976, № 4

X. KEPEC

\title{
ТЕНЗОРНЫЙ ГРАВИТАЦИОННЫЙ ПОТЕНЦИАЛ И ЧЕТЫРЕХМЕРНОЕ ПРЕДСТАВЛЕНИЕ НЬЮТОНОВСКОЙ ТЕОРИИ Э. КАРТАНОМ
}

\section{1. Введение}

Э. Картан $\left[{ }^{1}\right]$, А. Траутман $\left[{ }^{2}\right]$, П. Хавас $\left[{ }^{3}\right]$ и др. показали, что теория тяготения Ньютона является строгим предельным случаем теории Эйнштейна. Мы занимались этим вопросом с более практической точки зрения, имея в виду в первую очередь решение конкретных задач, например, физическую интерпретацию решений уравнений Эйнштейна $\left[{ }^{4}\right]$. Мы нашли, в отличие от других авторов, что нерелятивистский предел теории Эйнштейна выходит за рамки теории Ньютона, охватывая и теорию гравитационных полей неньютоновского типа, имеющих вихревой характер $\left[{ }^{5}\right]$. Релятивистские гравитационные поля могут быть изучены и классифицированы по свойствам их нерелятивистских пределов.

Э. Картан предложил модель искривленного пространства-времени, аффинная связность которого описывает ньютоновское гравитационное силовое поле. Ниже мы покажем элементарным способом, что аффинную связность картановской модели можно вывести из тензорного потенциала, который определяется с точностью до некоторых калибровочных преобразований.

П. Хавас рассмотрел возможность представления теории Ньютона в пространстве-времени, обладающем сингулярной метрикой. Упомянутый выше тензорный потенциал также определяет сингулярную метрику в пространстве-времени. Отсюда следует, что сингулярная метрика ньютоновского пространства-времени определяется не однозначно, а с точностью до калибровочных преобразований для тензорного потенциала.

\section{2. Модель Картана}

Пусть $e_{0}, e_{1}, e_{2}, e_{3}$ - векторы четырехмерного репера в пространствевремени и $\omega^{0}, \omega^{1}, \omega^{2}, \omega^{3}-$ компоненты инфинитезимального перемещения относительно этого репера. При перемещении $\omega^{\alpha(*)}$ репер $e_{\alpha}$ претерпевает изменение

$$
d e_{\alpha}=\omega_{\alpha}^{\sigma} e_{\sigma}
$$

* Греческие индексы пробегают значения $0,1,2,3$, а латинские $-1,2,3$. 
В пространстве без кручения линейные дифференциальные формы $\omega^{\alpha}, \omega_{\beta}^{\alpha}$ удовлетворяют уравнениям структуры

$$
\begin{aligned}
& d \wedge \omega^{\alpha}+\omega_{\sigma}^{\alpha} \wedge \omega^{\sigma}=0, \\
& d \wedge \omega_{\beta}^{\alpha}+\omega_{\sigma}^{\alpha} \wedge \omega_{\beta}^{\sigma}=\Omega_{\beta}^{\alpha} .
\end{aligned}
$$

Здесь формы $\Omega_{\beta}^{\alpha}$ характеризуют кривизну пространства.

Э. Картан показал, что для теории Ньютона характерны следующие соотношения:

$$
\begin{gathered}
\omega^{0}=d t, \quad \omega_{i}^{0}=0, \quad \omega_{i}^{k}+\omega_{k}^{i}=0, \\
\Omega_{i}^{k}=0, \quad \omega_{s} \wedge \Omega_{0}^{s}=0,
\end{gathered}
$$

$$
\omega^{2} \wedge \omega^{3} \wedge \Omega_{0}{ }_{0}+\omega^{3} \wedge \omega^{1} \wedge \Omega^{2}+\omega^{1} \wedge \omega^{2} \wedge \Omega_{0}^{3}=-4 \pi G \varrho \omega^{0} \wedge \omega^{1} \wedge \omega^{2} \wedge \omega^{3},
$$

где $\varrho$ - плотность вещества, порождающего поле. Если эти уравнения удовлетворены, существует прямоугольная декартова система координат $y^{i}$ такая, что

$$
\omega^{i}=d y^{i}, \quad \omega_{0}^{i}=-\Gamma^{i} d t, \quad \omega_{h}^{i}=0
$$

и далее

$$
\Gamma^{i}=\Phi_{, i}, \quad \Phi_{, s s}=-4 \pi G_{Q} .^{* *}
$$

Из (2) видно, что при $\varrho \neq 0$ все три формы $\Omega_{0}^{i}$ не могут обращаться в нуль одновременно. Следовательно, пространство-время искривлено.

\section{3. Введение сингулярной метрики}

В прямоугольной декартовой системе координат $y^{i}$, где гравитационное поле описывается скалярным потенциалом Ф, удовлетворяющим уравнению Пуассона, возьмем какое-то частное решение $\varphi\left(t, y^{i}\right)$ уравнения

$$
\varphi_{, 0}+1 / 2 \varphi_{, s} \varphi, s=\Phi \text {. }
$$

Затем определим для системы трех обыкновенных дифференциальных уравнений

$$
\dot{y}^{i}=\varphi_{, i}
$$

общее решение

$$
y^{i}=y^{i}\left(t, x^{1}, x^{2}, x^{3}\right),
$$

где $x^{1}, x^{2}, x^{3}$ - постоянные интегрирования. Внося функции (7) в уравнения (6) и дифференцируя по $t$, получаем, с учетом (5),

$$
\ddot{y}^{i}=\varphi, i 0+\varphi, i s \varphi, s=\Phi_{, i} \text {. }
$$

** Запятая обозначает обыкновенное дифференцирование; точка с запятой ковариантное дифференцирование относительно метрического тензора $\gamma_{\text {ik }}$ трехмерного пространства $t=$ const. 
Отсюда видно, что при любом выборе постоянных $x^{i}$ функции $y^{i}\left(t, x^{k}\right)$ являются координатами свободно падающей частицы в данном гравитационном поле. Следовательно, при переменных $x^{i}$ уравнения (7) определяют конгруэнцию мировых линий свободно падающих точек в некоторой конечной области пространства-времени. Такая конгруэнция представляет собой свободно падающее трехмерное пространство, каждой точке которого приписаны три координаты $x^{i}$. Будем называть его $x$-пространством.

Будем рассматривать, кроме свободно падающих $x$-пространств, еще ६-пространства произвольного типа, которые задаются уравнениями вида

$$
y^{i}=y^{i}\left(t, \xi^{1}, \xi^{2}, \xi^{3}\right),
$$

где правые части не подчинены никаким условиям, кроме условия дифференцируемости. Исключая $y^{i \cdot}$ из уравнений (7) и (8), получаем уравнения

$$
\xi^{i}=\xi^{i}\left(t, x^{1}, x^{2}, x^{3}\right),
$$

которые описывают движение точек $(x)$ свободно падающего $x$-про-

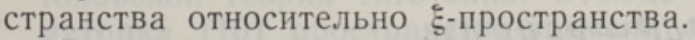

Рассмотрим две соседние точки, которые в момент $t+d t$ имеют координаты $\xi^{i}$ и $\xi^{i}+d \xi^{i}$. Первая точка покоится в $\xi$-пространстве, т. е. ее координаты $\xi^{i}$ постоянны; вторая точка покоится в свободно падающем $x$-пространстве, она движется относительно $\xi$-пространства со скоростью $u^{i}=\xi^{i}, 0$. Инфинитезимальный вектор, соединяющий эти точки, имеет в момент $t$ составляющие $d \xi^{i}-u^{i} d t$. Поэтому квадрат его длины в момент $t$ равен

$d s^{2}=\gamma_{r s}\left(d \xi^{r}-u^{r} d t\right)\left(d \xi^{s}-u^{s} d t\right)=u_{s} u^{s} d t^{2}-2 u_{s} d \xi^{s} d t+\gamma_{r s} d \xi^{r} d \xi^{s}$, где

$$
u_{i}=\gamma_{i s} u^{s}
$$

Если обозначить

$$
t=\xi^{0}, \quad u_{s} u^{s}=\gamma_{00}, \quad-u_{i}=\gamma_{i 0},
$$

можно записать

$$
d s^{2}=\gamma_{\mu \nu} d \xi^{\mu} d \xi^{\nu} .
$$

Очевидно, что $\operatorname{det}\left(\gamma_{\alpha \beta}\right)=0$.

Покажем, что квадратичная форма (10) инвариантна относительно преобразований

$$
\xi^{i}=f^{i}\left(t, \bar{\xi}^{1}, \bar{\xi}^{2}, \bar{\xi}^{3}\right), \quad t=\bar{t},
$$

которые являются самыми общими преобразованиями координат, допустимыми в теории Ньютона. Действительно, мы имеем

$$
\begin{aligned}
& d \xi^{i}=f^{i}, 0 d t-1-f^{i},{ }_{, s} d \bar{\xi}^{s}=u^{i} d t+\xi^{i}, p d x^{p}, \\
& \overline{d \xi^{i}}=\bar{u}^{i} d t-1-\bar{\xi}^{i}, p d x^{p},
\end{aligned}
$$

где $\bar{u}^{i}$ - скорость свободно падающего $x$-пространства относительно $\bar{\xi}$-пространства, Из этих соотношений находим

$$
d \xi^{i}-u^{i} d t=f^{i},{ }_{, s}\left(\bar{d}^{s}-\bar{u}^{s} d t\right) .
$$


Подставляя это выражение в равенство (10), получаем

$$
d s^{2}=\bar{\gamma}_{p q}\left(\bar{d}^{p}-\bar{u}^{p} d t\right)\left(\bar{d}^{q}-\bar{u}^{q} d t\right),
$$

где

$$
\bar{\gamma}_{p q}=f^{r}, p f^{s}, q \gamma_{r s} .
$$

Из доказанной инвариантности следует, что квадратичная форма (10) определяет метрику в пространстве-времени, сингулярную в смысле П. Хаваса.

\section{4. Тензорный потенциал}

В силу равенства $\operatorname{det}\left(\gamma_{\alpha \beta}\right)=0$ невозможно вычислить контравариантные компоненты метрического тензора по формуле $\gamma_{\alpha \sigma} \gamma^{\beta \sigma}=\delta_{\alpha}^{\beta}$. Эта трудность преодолима следующим образом.

Линейный элемент (10) имеет свое подобие в общей теории относительности. Здесь свободно падающее $x$-пространство определяется координатной системой, в которой

$$
d s^{2}=c^{2} d t^{2}-g_{r s} d x^{r} d x^{s} .
$$

В произвольном छ-пространстве, определенном уравнениями (9), имеем

$$
-d s^{2}=\left(-c^{2}+u_{s} u^{s}\right) d t^{2}-2 u_{s} d \xi^{s} d t+\gamma_{r s} d \xi^{r} d \xi^{s},
$$

где

$$
u_{i}=\gamma_{i s} u^{s}, \quad g_{i k}=\gamma_{r s} \xi_{i} \xi_{k} \xi_{k},
$$

и $u^{i}$ - скорость свободно падающего $x$-пространства относительно $\xi$-пространства. Видим, что коэффициенты квадратичной формы (11) имеют то же кинематическое значение, что и коэффициенты формы (10). Более того, если перейти формально к пределу при $c \rightarrow \infty$ и в то же время пренебречь бесконечным членом $c^{2} d t^{2}$, то релятивистская фундаментальная форма (11) перейдет в форму (10). При этом контравариантные компоненты метрического тензора (11) переходят в контравариантные компоненты тензора (10).

Воспользуемся этим обстоятельством. Прибавим сначала к правой части равенства (10) член $-c^{2} d t^{2}$, затем вычислим составляющие $\gamma^{\alpha \beta}(c)$ и перейдем к пределу при $c \rightarrow \infty$. Получим контравариантные компоненты сингулярного метрического тензора (10) в следующем виде:

$$
\gamma^{00}=\gamma^{i 0}=0, \quad \gamma^{i k},
$$

где $\gamma^{i k}$ определяются из соотношения $\gamma_{i s} \gamma^{k s}=\delta_{i}^{k}$.

Аналогичным образом вычислим компоненты аффинной связности, или, что то же самое, линейные дифференциальные формы

$$
\omega_{\beta}^{\alpha}=\Gamma_{\beta \sigma}^{\alpha} \omega^{\sigma}, \quad \omega^{0}=d t, \quad \omega^{i}=d \xi^{i}
$$

для сингулярной метрики. Получим следующие выражения:

$$
\begin{aligned}
& \omega_{0}^{0}=\omega_{i}^{0}=0, \\
& \omega_{0}^{i}=-\Gamma^{i} d t+\Gamma_{s}^{i} d \xi^{s}, \\
& \omega_{k}^{i}=\Gamma_{k}^{i} d t+\Gamma_{k s}^{i} d \xi^{s},
\end{aligned}
$$


где

$$
\begin{array}{ll}
\Gamma^{i}=\gamma^{i s} \Gamma_{s}, & \Gamma_{i}=u_{i, 0}-1 / 2\left(u_{s} u^{s}\right)_{, i}, \\
\Gamma_{k}^{i}=\gamma^{i s} \Gamma_{k s}, & \Gamma_{k i}=1 / 2\left(\gamma_{k i, 0}+u_{k, i}-u_{i, k}\right), \\
\Gamma_{k j}^{i}=\gamma^{i s} \Gamma_{k j, s}, & \Gamma_{k j, i}=1 / 2\left(\gamma_{j i, k}+\gamma_{k i, j}-\gamma_{k j, i}\right) .
\end{array}
$$

В прямоугольной декартовой системе координат $y^{i}$ имеем

$$
\gamma_{i k}=\delta_{i k}, \quad u_{i}=\varphi, i,
$$

вследствие чего

$$
\Gamma_{k}^{i}=0, \quad \Gamma_{k j}^{i}=0, \quad \Gamma_{i}=[\varphi, 0+1 / 2(\varphi, s \varphi, s)]_{, i}=\Phi_{, i},
$$

так что

$$
\omega^{0}=d t, \quad \omega^{i}=d y^{i}, \quad \omega_{0}^{i}=-\Phi_{, i} d t, \quad \omega_{k}^{i}=0
$$

Это и есть картановские соотношения (3) и (4), характерные для ньютоновской теории тяготения. Отсюда мы заключаем, что сингулярная метрика (10) пространства-времени описывает ньютоновское гравитационное поле. Коэффициенты $u_{i}, \gamma_{i k}$ - их девять - переменные поля. Они являются компонентами тензора второго ранга относительно группы общих преобразований координат в теории Ньютона. Это - ньютоновский гравитационный потенциал. Квадратичная дифференциальная форма (10) может быть рассмотрена как потенциал для картановских линейных дифференциальных форм $\omega_{\alpha}^{\beta}$.

\section{5. Калибровочные преобразования}

В римановом пространстве с метрикой (11) уравнения геодезических имеют вид

$$
d V^{\alpha}+\omega_{\sigma}^{\alpha} V^{\sigma}=0
$$

где

$$
V^{0}=\frac{d t}{d s}=\frac{1}{c \beta}, \quad V^{i}=\frac{d \xi^{i}}{d s}=\frac{\dot{\xi}^{i}}{c \beta},
$$

причем

$$
c^{2} \beta^{2}=c^{2}-\gamma_{r s}\left(\dot{\xi} r-u^{r}\right)\left(\dot{\xi} s-u^{s}\right) .
$$

Переходя к пределу при $c \rightarrow \infty$ и учитывая, что $\beta \rightarrow 1$ и что согласно формулам (12) вдоль кривой имеет место

$$
\begin{aligned}
& \omega_{0}^{i}=\left(-\Gamma^{i}+\Gamma_{s}^{i} \dot{\xi}^{s}\right) d t, \\
& \omega_{k}^{i}=\left(\Gamma_{k}^{i}+\Gamma_{k s}^{i} \dot{\xi}^{s}\right) d t,
\end{aligned}
$$

получаем уравнения геодезических для сингулярной метрики (10) в следующей форме:

$$
\ddot{\xi}^{i}+\Gamma_{r s}^{i} \dot{\xi} \dot{\xi}^{s}-\Gamma^{i}+2 \Gamma_{s}^{i} \dot{\xi}^{s}=0 .
$$


Целесообразно переписать их в виде

$$
\frac{d}{d t}\left(\gamma_{i s} \dot{\xi}^{s}\right)-1 / 2 \gamma_{r s, i} \dot{\xi} \dot{\xi}^{s}=\Gamma_{i}+2 \omega_{i s} \dot{\xi},
$$

где

$$
\omega_{i k}=1 / 2\left(u_{i, k}-u_{k, i}\right)
$$

В прямоугольной декартовой системе координат $y^{i}$, где $\gamma_{i k}=\delta_{i k}$, $\Gamma_{i}=\Phi_{, i}$ и $2 \omega_{i k}=\varphi_{, i k}-\varphi, k i=0$, уравнения (13) принимают вид

$$
\ddot{y}^{i}=\Phi_{, i} \text {. }
$$

Отсюда видно, что уравнения геодезических (13) описывают свободное

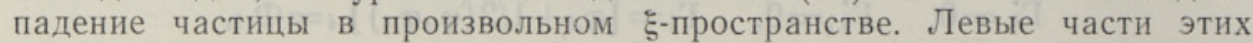
уравнений представляют собой компоненты ускорения частицы, а правые части - компоненты гравитационных сил, действующих на частицу. Гравитационные силы выводятся из тензорного потенциала по формулам

$$
\begin{aligned}
\Gamma_{i} & =u_{i, 0}+1 / 2\left(u_{s} u^{s}\right)_{, i}, \\
\omega_{i k} & =1 / 2\left(u_{i, k}-u_{k, i}\right) .
\end{aligned}
$$

Видим, что кроме силы притяжения $\Gamma_{i}$, существует еще сила $2 \omega_{i s} \dot{\varepsilon}^{8}$ типа силы Кориолиса. Последняя исчезает в $y$-пространстве, где закон свободного падения имеет вид (14). Теперь мы в состоянии проследить, в какой степени тензорный гравитационный потенциал определен однозначно и каким преобразованиям он подвержен в данном छ-пространстве. Эти преобразования будем называть калибровочными.

Мы определили квадратичную форму (10) с помощью произвольного $\xi$-пространства и некоторого базисного свободно падающего $x$-пространства. Мы показали, что при переходе из одного ६-пространства в другое, но при неизменном $x$-пространстве, квадратичная форма (10) не изменяется. Однако дело обстоит иначе, если при неизменном странстве заменить базисное $x$-пространство новым свободно падающим $\bar{x}$-пространством, т. е. если при определении $x$-пространства исходить из другого частного решения $\bar{\varphi}\left(t, y^{i}\right)$ для уравнения $(5)$. Тогда будем иметь в $\xi$-пространстве два набора полевых переменных: $u_{i}, \gamma_{i k}$ и $\bar{u}_{i}$, $\gamma_{i k}$, где $u^{i}$ обозначает скорость $x$-пространства относительно ства, а $\bar{u}^{i}-$ скорость $\bar{x}$-пространства. Закон свободного падения (13) может быть сформулирован равным образом как с помощью переменных $u_{i}, \gamma_{i k}$, так и с помощью переменных $\bar{u}_{i}, \bar{\gamma}_{i k}$. Поскольку левая часть уравнения (13) не зависит от $u_{i}$, то должно иметь место равенство

$$
\Gamma_{i}+2 \omega_{i s} \dot{\xi}^{s}=\bar{\Gamma}_{i}+2 \bar{\omega}_{i s} \dot{\xi}^{s},
$$

или, учитывая формулы (15),

$$
\begin{aligned}
u_{i, 0}+1 / 2\left(u_{s} u^{s}\right)_{, i} & =\bar{u}_{i, 0}+1 / 2\left(\bar{u}_{s} \bar{u}^{s}\right)_{, i}, \\
u_{i, k}-u_{k, i} & =\bar{u}_{i, k}-\bar{u}_{k, i} .
\end{aligned}
$$

Из последнего уравнения вытекает

$$
\bar{u}_{i}-u_{i}=\psi_{, i},
$$

а первое уравнение налагает на функцию $\psi$ условие

$$
\psi, 0+1 / 2 \gamma^{r s} \psi, r\left(\psi, s+2 u_{s}\right)=0
$$


Формулы (16) и (17) определяют калибровочное преобразование тензорного гравитационного потенциала при переходе к новому базисному свободно падающему $x$-пространству. Силы тяготения $\Gamma_{i}$ и $2 \omega_{i s} \dot{\xi}^{s}$ инвариантны относительно калибровочных преобразований. Нетрудно убедиться, что фундаментальная квадратичная форма (10) преобразуется по формуле

$$
d \bar{s}^{2}=d s^{2}+2 d \psi d t
$$

Для заданного гравитационного поля метрика ньютоновского пространства-времени определяется неоднозначно.

\section{6. Уравнения поля и вихревые гравитационные поля}

Уравнения поля, которым должен удовлетворять тензорный гравитационный потенциал, получаются из картановских соотношений (1) и (2). Встречающиеся в них внешние дифференциальные формы

$$
\Omega_{\beta}^{\alpha}=1 / 2 R \alpha_{\beta \rho \sigma \omega}^{\rho} \wedge \omega^{\sigma},
$$

коэффициентами которых являются компоненты тензора кривизны, следует выразить через переменные поля. Применяя вышеописанный метод предельного перехода и используя второе уравнение структуры, находим для сингулярной метрики (10) следующие выражения:

$$
\begin{aligned}
& \Omega_{0}^{0}=\Omega_{i}^{0}=0, \\
& \Omega_{0}^{i}=\left(\Gamma_{; s}^{i}+\Gamma_{s, 0}^{i}+\Gamma_{s}^{p} \Gamma_{p^{i}}\right) d t \wedge d \xi^{s}+\Gamma_{s ; r}^{i} d \xi^{r} \wedge d \xi^{s}, \\
& \Omega_{h}^{i}=\left(\Gamma_{k s, 0}^{i}-\Gamma_{h ; s}^{i}\right) d t \wedge d \xi^{s}+\left(\Gamma_{k s, r}^{i}+\Gamma_{p r}^{i} \Gamma_{k s}^{p}\right) d \xi^{r} \wedge d \xi^{s} .
\end{aligned}
$$

Из картановского условия $\Omega_{k}^{i}=0$ вытекает

$$
\begin{gathered}
\Gamma_{k j, 0}^{i}-\Gamma_{h ; j}^{i}=0, \\
\Gamma_{h j, h}^{i}-\Gamma_{k h, j}^{i}+\Gamma_{s h}^{i} \Gamma_{h j}^{s}-\Gamma_{s j}^{i} \Gamma_{k h}^{s}=0 .
\end{gathered}
$$

В левой части последнего уравнения стоит тензор кривизны трехмерного пространства $t=$ const. Как известно, в трехмерном случае исчезновение тензора Риччи $P_{i k}$ влечет за собой исчезновение тензора кривизны, и наоборот. Ввиду этого вместо уравнения (20) можно записать

$$
P_{i k}=0 .
$$

Уравнение (19) равносильно уравнению

$$
\Gamma_{k ; j}^{i}-\Gamma_{j ; k}^{i}=0,
$$

как это видно из тождества

$$
\gamma_{i s} \Gamma_{j k, 0}^{s} \equiv \Gamma_{i k ; j}-\Gamma_{j k ; i}+\Gamma_{j i ; k} .
$$

В силу уравнения (21) второй член в выражении $\Omega_{0}^{i}$ обращается в нуль, так что остается

$$
\Omega_{0}^{i}=\left(\Gamma_{; 3}^{i}+\Gamma_{s, 0}^{i}+\Gamma_{s} p \Gamma_{p}^{i}\right) d t \wedge d \xi^{s} .
$$


Если подставить это выражение в условие $\omega_{s} \wedge \Omega_{0}^{s}=0$, получим тождество. Наконец, условие (2) дает уравнение

$$
\Gamma_{s, 0}^{s}+\Gamma_{r}^{s} \Gamma_{s}^{r}+\Gamma_{; s}^{s}=-4 \pi G_{Q} .
$$

Таким образом, из условий Картана (1) и (2) вытекают следующие уравнения поля теории Ньютона:

$$
\begin{gathered}
P_{i k}=0, \\
\Gamma_{k i ; j}-\Gamma_{j i ; k}=0, \\
\Gamma_{s, 0}^{s}+\Gamma_{s}^{r} \Gamma_{r}^{s}+\Gamma_{; s}^{s}=-4 \pi G_{\varrho} .
\end{gathered}
$$

Если подставить выражения

$$
\Omega_{\beta}^{\alpha_{i}}=1 / 2 R_{\beta \rho \sigma}^{\alpha^{\rho}} \wedge \omega^{\sigma}
$$

в соотношения (1) и (2), получим уравнения поля в такой форме:

$$
\begin{aligned}
& R^{i}{ }_{k 0 j}=0, \\
& R^{i}{ }_{k h j}=0, \\
& R^{s_{00 s}}=-4 \pi G_{\varrho} .
\end{aligned}
$$

Мы знаем, что уравнение (24) эквивалентно уравнению

$$
P_{i k}=R_{i k s}=0 \text {. }
$$

Учитывая, кроме того, что левая часть уравнения (25), очевидно, равна $R^{\sigma}{ }_{0 \sigma}=R_{00}$, можем уравнения $(23)-(25)$ переписать в виде

$$
R_{00}=-4 \pi G_{Q}, \quad R^{i_{k 0 j}}=0, \quad P_{i k}=0 .
$$

В то же время можно показать, что при предельном переходе $c \rightarrow \infty$ уравнения Эйнштейна переходят не в уравнения (26), а в более общие нерелятивистские уравнения

$$
R_{00}=-4 \pi G_{\varrho}, \quad R_{i 0}=0, \quad P_{i k}=0 .
$$

Это значит, что теория Эйнштейна в нерелятивистском приближении шире теории Ньютона. Теория гравитации, основанная на уравнениях (27), описывает и гравитационные поля неньютоновского типа - т. н. вихревые гравитационные поля. Для последних характерно то обстоятельство, что в них, в отличие от гравитационных полей ньютоновского типа, гравитационные силы типа сил Кориолиса не могут быть устранены никаким выбором системы прямоугольных декартовых координат $y^{i}$.

Заметим, что уравнения (26) и (27) есть тензорные уравнения относительно группы общих преобразований координат для ньютоновской теории.

\section{7. Тензорный потенциал вихревого гравитационного поля}

Для гравитационного поля ньютоновского типа существует прямоугольная декартова система координат $y^{i}$, где поле описывается скалярным потенциалом $\Phi\left(t, y^{i}\right)$, удовлетворяющим уравнению Пуассона. В такой координатной системе фундаментальная форма (19) принимает вид

$$
d s^{2}=2 \Phi d t^{2}-2 d \varphi d t+d y^{s} d y^{s}
$$


В этом легко убедиться, имея в виду, что $u_{i}=\varphi, i$ и скаляр $\varphi$ удовлетворяет уравнению (5).

Вихревое гравитационное поле описывается двумя скалярными функциями $\Phi\left(t, y^{i}\right)$ и $\psi\left(t, y^{i}\right)$, первая из которых удовлетворяет уравнению Пуассона, а вторая - уравнению Лапласа, причем не все вторые производные $\psi, i k$ равны нулю $\left[{ }^{5}\right]$.

Пусть $u^{k}\left(t, y^{i}\right)$ - скорость свободно падающего $x$-пространства относительно $y$-пространства. Для гравитационного поля ньютоновского типа мы могли написать $u^{i}=\varphi, i$, что является следствием из полевого уравнения $R^{i}{ }_{k 0 j}=0$. В вихревом гравитационном поле мы полагаем

$$
u^{i}=\varphi_{, i}-\varepsilon_{i r s} a^{r}, s .
$$

При заданных $\Phi$ и $\psi$ скаляр $\varphi$ и вектор $a^{i}$ определяются из системы четырех уравнений

$$
\begin{array}{r}
a^{i}{ }_{, s s}-a^{s}{ }_{, s i}=2 \psi, i, \\
\varphi, 0+1 / 2 u^{s} u^{s}=\Phi+\psi^{2} .
\end{array}
$$

В силу этих уравнений в координатной системе $y^{i}$ фундаментальную форму (10) можно выразить в виде

$$
d s^{2}=2\left(\Phi+\psi^{2}\right) d t^{2}-2 d \varphi d t+2 \varepsilon_{s p q} a^{p}{ }_{, q} d y^{s} d t+d y^{8} d y^{s} .
$$

Из (29) и (30) вытекает следующий закон свободного падения:

$$
\ddot{y}_{i}=\dot{u}^{i}=\left(\Phi+\psi^{2}\right)_{, i}+2 \varepsilon_{i r s} u^{r} \psi_{, s}-\varepsilon_{i r s} a^{r}, s 0 .
$$

Это - уравнения геодезических для сингулярной метрики (31).

Вихревое гравитационное поле может быть представлено картановской моделью, если в ней условие $\Omega_{i}^{k}=0$ заменить соотношениями

$$
\begin{gathered}
\omega^{0} \wedge \omega^{k} \wedge \Omega_{i}^{j}-\omega^{0} \wedge \omega^{j} \wedge \Omega_{i}^{k}=0 \\
\omega^{2} \wedge \omega^{3} \wedge \Omega_{i}^{1}+\omega^{3} \wedge \omega^{1} \wedge \Omega_{i}^{2}+\omega^{1} \wedge \omega^{2} \wedge \Omega_{i}^{3}=0 .
\end{gathered}
$$

ЛИТЕРА Т РА

1. C a rt a n E., Ann. de L'Ecole Normale sup., 40, 325 (1923); 41, 1 (1924).

2. Tr a u tm a n A., C. R., 257, 617 (1963).

3. H a v a s P., Revs Mod. Phys., 36, 938 (1964).

4. К е р е с Х., ЖЭТФ, 52, 768 (1967).

5. К е р е х., ЖЭТФ, 48, 1320 (1965).

$\begin{array}{cc}\text { Институт физики } & \text { Поступила в редакцию } \\ \text { Академии наук Эстонской ССР } & 1 / \mathrm{III} 1976\end{array}$

H. KERES

\section{GRAVITATSIOONIVÄLJA TENSORPOTENTSIAAL JA NEWTONI TEOORIA CARTANI NELJADIMENSIOONILISES ESITUSES}

Vaadeldakse üht Newtoni aegruumi singulaarse meetrika defineerimise elementaarset viisi. Võrdlus Newtoni gravitatsiooniteooria neljadimensioonilise esitusega, mille autor on E. Cartan, näitab, et mainitud singulaarne meetriline tensor on vaadeldav gravitatsioonivälja tensorpotentsiaalina, mis on määratud teatud kalibreerimisteisenduste täpsuseni. Ta rahuldab võrrandeid, mis kujutavad erijuhtu Einsteini võrrandeist piir- 
juhul $c \rightarrow \infty$. Gravitatsiooniteooria, mis tugineb viimati mainitud piirjuhulistele Einsteini võrrandeile, kirjeldab peale Newtoni tüüpi gravitatsiooniväljade veel pöörisvälju, mida iseloomustab fakt, et neis väljades ei kao Coriolise jōu tüüpi gravitatsioonijõud mitte üheski täisnurkses karteesiuse koordinaadisüsteemis.

\section{H. KERES}

\section{THE GRAVITATIONAL TENSOR POTENTIAL AND CARTAN'S FOUR- DIMENSIONAL REPRESENTATION OF NEWTONIAN THEORY}

An elementary method of introduction of the Newtonian space-time metric singular in the sense of P. Havas is considered. Comparison with Cartan's four-dimensional model of the Newtonian theory of gravitation shows that the singular metric tensor mentioned above plays the role of the gravitational tensor potential determined uniquely up to certain gauge transformations. It satisfies the field equations which are a special case of Einstein's equations taken in their non-relativistic limit as $c \rightarrow \infty$. A gravitational theory based upon these limiting equations describes, besides Newtonian gravitational fields, also rotational gravitational fields which are characterized by the iact that the Coriolis force type gravitational forces cannot be removed by any choice of rectangular Cartesian coordinate system. The tensor potential of rotational gravitational field is determined, and the modifications of Cartan's model due to rotational gravitational fields are given. 